\title{
Non-invasive IoT sensing and monitoring system for neonatal care
}

\author{
José Ilton de Oliveira Filho ${ }^{1}$, Otacilio da Mota Almeida ${ }^{2}$, Nadya Yousef ${ }^{3}$ and Khaled Nabil Salama ${ }^{1}$ \\ ${ }^{1}$ King Abdullah University of Science and Technology, Thuwal, Saudi Arabia \\ ${ }^{2}$ Universidade Federal do Piauí, Teresina, Brazil \\ ${ }^{3}$ Division of Pediatrics and Neonatal Critical Care, APHP - Paris Saclay University Hospitals, "A. Béclère” Medical center, Paris, \\ France
}

\begin{abstract}
The scientific innovation in health systems moves towards non-invasive and wearable approaches to acquire and measure vital signs. Due to its non-invasive nature, infrared (IR) thermography has become the preferred choice for monitoring physiological signals from a distance. Combining IR non-invasive monitoring with the Internet of Things (IoT), we developed a risk management system for neonatal incubators, which has the capability to inform temperature anomalies from the newborn, aiming to prevent or to have a fast-responding action to risk situations such as hypothermia and hyperthermia. With multiarea-temperature monitoring we can detect signs of hypothermia minutes before decrease in core temperature. As a result, the system can record a thermal image from inside the incubator every 0.5 seconds, upload to the cloud every 20 seconds, perform real-time data analysis, activate alarms of possible risk-eminent situations and send notifications with maximum delay of 2 minutes.
\end{abstract}

Keywords - Measurement; Neonatal; Infrared; Thermal Imaging; Newborn; Iot; E-Health; Monitoring; Non-invasive;

\section{INTRODUCTION}

The neonatal period is the most vulnerable period for child survival and a critical phase for later development [1]. Hypothermia remains an important contributor to neonatal mortality worldwide [2]. Maintaining a normal body temperature (BT) is thus a critical function for the survival of the newborn baby (NB). However, especially for preterm, lowbirth weight, and sick NBs, mechanisms for temperature regulation may easily be overwhelmed, leading to hypothermia, cold stress, metabolic deterioration, and, ultimately, death [2]. Improving temperature stability for sick and preterm infants, therefore, continues to be an area of priority in the neonatal intensive care unit (NICU).

The very preterm infant ( $<32$ weeks) and the very low birth weight infants (VLBW) have immature mechanisms for thermoregulation and cannot maintain BT without an additional, external heat source. Keeping the BT within a narrow target range is challenging since these vulnerable newborns have a high body surface to weight ratio, immature temperature control mechanisms, and a large metabolic cost of temperature control [3-5]. Temperature instability may also be an early sign of sepsis. Detection of abnormal temperatures is, therefore, essential to ensure timely intervention and treatment. Continuous monitoring and control of the body temperature is thus a cornerstone of neonatal care.
In addition, a calm and comfortable environment is essential to the NB's wellbeing, and disturbing influences may inhibit the physiological stability, recovery, and growth of fragile infants. Measurement techniques and alarm systems must respect the NB's fragility and high sensitivity to environmental stimuli to improve outcomes.

Infrared thermography, also known as Medical Infrared Therapy (MIT), is a non-invasive and non-irradiating technique for temperature measurement that does not require direct handling of the infant [6]. MIT is used to detect and localize thermal abnormalities characterized by an abnormal increase or decrease of the temperature of the skin surface. The method consists of the detection of infrared radiation that can be directly correlated with the temperature of a defined body region [6]. The heat loss of NB can be calculated through the emitted radiation inside the incubator enclosure, as illustrated in Fig. 1.

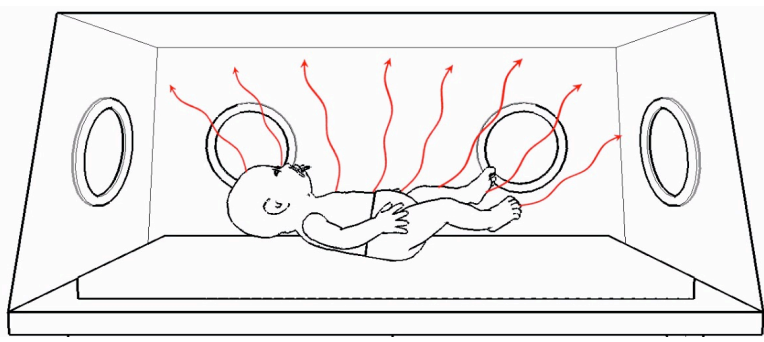

Fig. 1. IR radiation emitted by an NB inside a neonatal incubator.

The present work aims to evaluate an MIT-based noninvasive system to analyze and monitor neonatal BT, triggering warnings and alarms, if the resulting BT values go beyond a defined target range. This work also seeks to automate and improve the acquisition of physiological parameters to decrease the need for temperature-related handling of infants, thereby enhancing comfort for NICU-admitted babies.

\section{RELATED WORK AND BASIC BACKGROUND}

The merging of cloud technology and the Internet of Things (IoT) has facilitated works about the development of more efficient and comfortable monitoring systems [7]. Research on non-invasive and IoT for monitoring physical conditions explore a wide range of technics, such as reflectance pulse oximeter [8, 9], laser doppler methods [10], digital stethoscope [11], near-field communication (NFC) sensing 
[12], camera-based photoplethysmography [13-16], piezoelectric sensors [17-19], capacitive sensors [20, 21], and transcutaneous electromyography [22].

The non-invasive nature of infrared thermography (IR) makes it a preferred choice for the monitoring of physiological signals without any direct contact with the NB. The concept Neonatal Infrared Imaging (NIRT) was introduced in 1980 by Clark and Stothers, who performed the first measurements in an NICU [23]. In 2000, Adams et al. [24] achieved successfully direct thermography and determined the energy expenditure in preterm infants by monitoring NBs inside a convective incubator, kangaroo mother care, and open radiant warmer. Many other studies have demonstrated the application of thermography in medicine as a method for investigating thermoregulation and necrotizing agents in preterm newborns [23-27].

An infrared thermography system works through the concept that every physical body, which has temperature different of absolute zero, loses heat predominantly by the emission of the infrared electromagnetic radiation spectrum. This spectrum can be detected, and from it, the heat can be estimated. In a NICU, the heat loss and the radiation emitted depends on the exposed skin area, the temperature of the surfaces that enclose the newborn and the temperature of the environment [28].

\section{System Description}

To ensure a full-body skin temperature, the sensor is positioned at the top of the incubator, and the shutter is placed in a small hole made in the acrylic hood. The full-body image is essential to identify the current step of NB's thermal regulation in each part of the body. Fig. 2 presents the projection of the prototype in use.

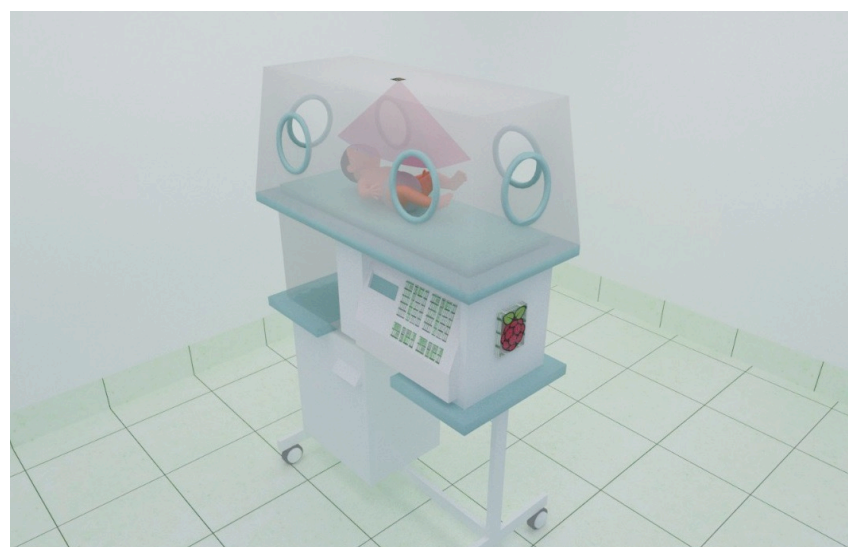

Fig. 2. Sketch of the system in operation.

The proposed system diagram can be visualized in Fig. 3 . The system uses as a central component the long wave infrared (LWIR) array sensor Lepton Flir to acquire 160x120 values of temperature from inside of the neonatal incubator. Those values are sent to a Raspberry $\mathrm{Pi}$, which processes it and uploads the data to the cloud to be stored, displayed, and used to trigger notifications to any smartphone connected to the system. If any abnormality in the temperature is detected, alarms, notifications, and alerts can be triggered depending on the significance of the anomaly.

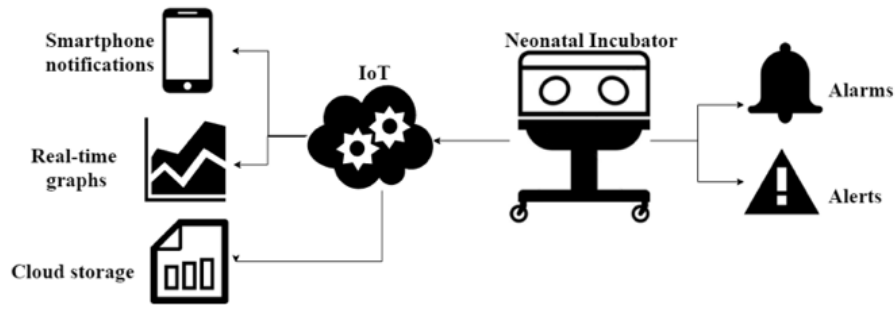

Fig. 3. Diagram of the developed system.

The Raspberry Pi, operating with a Raspibian Linux system, obtain the raw data through SPI connection and processes it creating two different files. The first file created is a thermo-image, and the second one is a text file containing the raw data provided by the sensor. After generating those files, the Raspberry identifies the object of interest (OI) and its average temperature and classifies warmers and cold areas. After been processed, the data is sent to the IFTT integrated services.

When the system detects any environmental risk, which can generate consequences to the health of the baby, alarms and alerts are activated in order to prevent health complications. Alerts are triggered by small anomalies that can represent minors' risk for the newborn. When alerts are triggered, the system generates sounds and blink lights. Alarms are triggered by risk situations that can have significant impact on the life of the neonate. When alarms are triggered, the system sends smartphone notifications, generate sounds and blink small lights. The alerts and alarms are presented in Table I below.

TABLE I. ALERTS, ALERTS AND TRIGGER CONDITIONS

\begin{tabular}{|c|c|}
\hline Alerts & Trigger Conditions \\
\hline Alert-1 & Abrupt temperature change \\
\hline Alert-2 & High-temperature region \\
\hline Alert-3 & Low-temperature region \\
\hline Alert-4 & $\begin{array}{l}\text { More than one object in the } \\
\text { incubator }\end{array}$ \\
\hline Alert-5 & No object detected \\
\hline Alarms & Trigger Conditions \\
\hline $\begin{array}{c}1 \text { - cold stress (mild hypothermia) } \\
2 \text { - Moderate hypothermia }\end{array}$ & $\begin{array}{l}\text { temperature between } 36.0 \\
\text { and } 36.4^{\circ} \mathrm{C} \\
\text { temperature between } 32.0 \\
\text { and } 35.9^{\circ} \mathrm{C}\end{array}$ \\
\hline 3 - Severe Hypothermia & temperature less than $32.0^{\circ} \mathrm{C}$ \\
\hline 4 - Hyperthermia & temperature above $37.5^{\circ} \mathrm{C}$ \\
\hline
\end{tabular}

The notifications are generated by the IoT service IFTTT. The IFTTT is a WEB service that helps to connect supplementary WEB services. The IFTTT does not process any data, only serves as a bridge to connect other applications, combining their tasks to perform a particular function [29]. In this project, the IFTTT was combined with the Photon Particle (P0) device to read the data in the cloud sent by the Raspberry. 
In short, the camera Lepton sends to the Raspberry raw data which are processed and sent to ThingSpeak cloud storage service, from there, the Photon read the data and triggers notification in the smartphones by IFTT.

\section{RESULTS}

The experimental results were obtained in the laboratory, without measurements on real NB. Resistive charges were placed inside the neonatal incubator and tested using an adult human hand as a model. Fig. 4 shows a picture of the system inside the incubator.

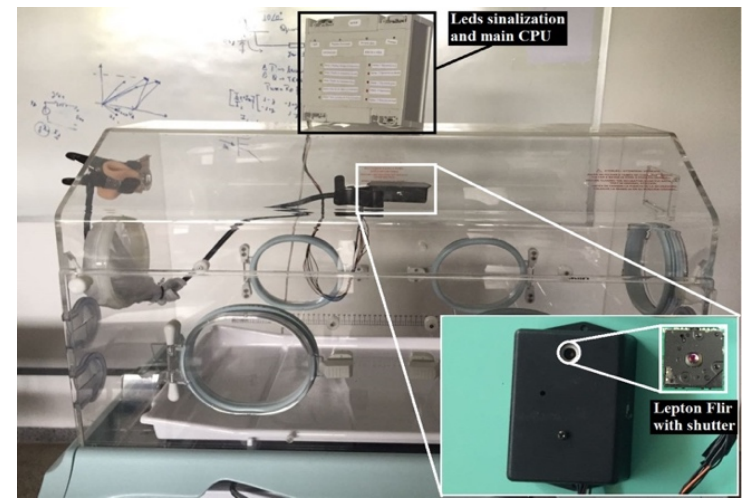

Fig. 4. The system installed inside of the incubator for calibration.

The thermographic image acquired by the camera has a resolution of $160 \times 120$ pixels and features sharpness, high uniformity, easy objects perception, and differentiation in the image. The original image from the inside of the neonatal incubator is shown in Fig 5.A; the points of maximum and minimum value in 5.B; the object of interest in 5.C; In Fig 5.D the areas with the maximum temperature value of the image are shown, in dark red, while the regions with temperature slightly above the average temperature are shown in light red; Fig. 5.E shows the areas with the highest temperature difference from the average.

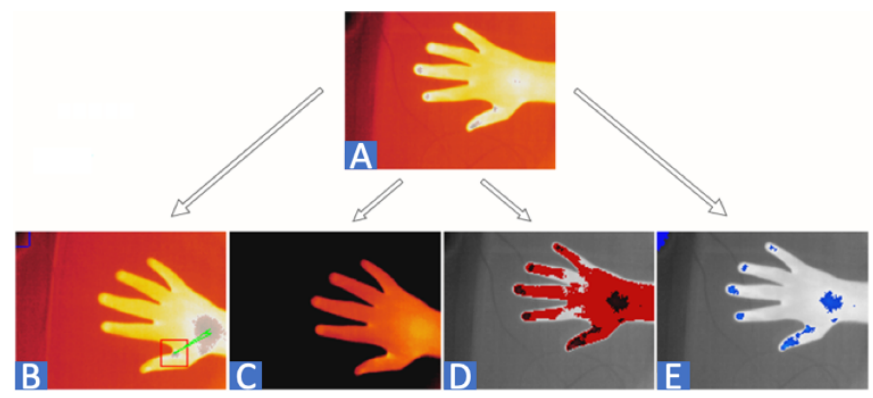

Fig. 5. Thermal image and its outcomes after different processing.

The temperature data obtained by the Lepton were sent to the ThingSpeak platform, where they were stored and were plotted for better visualization, using the online application of MathWorks. The tests were conducted with resistive loads inside the incubator, and the minimum, average, and maximum temperature were measured every 0.5 seconds and sent to the cloud platform every 20 seconds. Only the maximum temperature data were plotted and used to trigger the alarms.
The temperature decreasing graph is shown in Fig. 6 A. The events 1, 2, 3, and 4 are the moment when the value goes below the normal temperature range, characterizing hypothermia. The notifications referent to events 1 to 4 are presented in Fig. $6 \mathrm{~B}$ and $\mathrm{C}$.

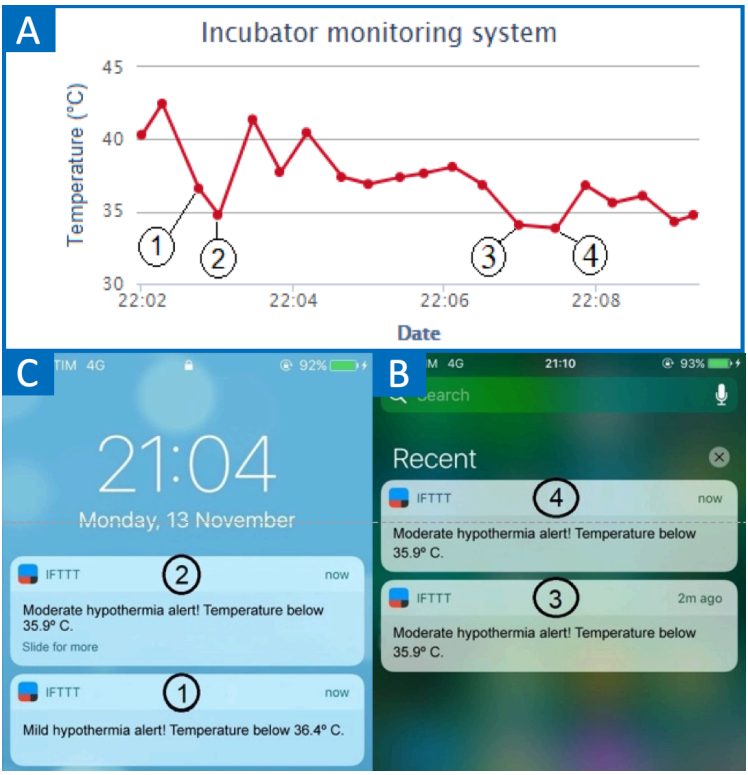

Fig. 6. A) Temperature decreasing chart and notifications received due to events B) 1,2 and C) 3 and 4 .

Note that the notification occurred in less than 2 minutes of the events, but due to the daylight saving mode of the Raspberry, an "artificial" 1-hour difference is shown. The temperature increasing graph is depicted in Fig. 7.A. Events 5 and 6 are shown in Fig. 7.A and represent events when the recorded temperature increased above the defined limit. The notifications due to this event and its graphs can be seen in Fig. $7 \mathrm{~B}$ and $\mathrm{C}$.

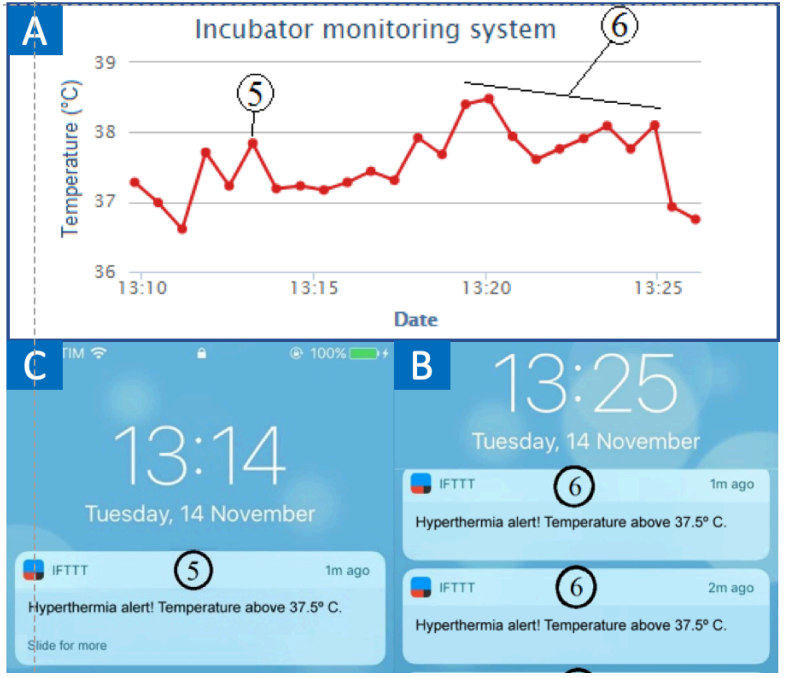

Fig. 7. A)Temperature increasing chart with notifications received due event B) 5 and C) 6 . 


\section{CONCLUSION}

In conclusion, we demonstrated a fully working system that can be integrated with a neonatal incubator to access its internal thermal distribution. The system has the capability to segment the captured thermal distribution into four different thermal images. Each of these images has a function to identify cold and warm temperature regions and their boundaries. This system monitors the abrupt changes in the temperature spatially while saving the data on a cloud WEB service. Besides this temperature monitoring capability, the system can also send notifications to user's smartphone in case of an event that can result in a risk for the neonate.

\section{REFERENCES}

[1] W. H. Organization, "Every newborn: an action plan to end preventable deaths," 2014.

[2] K. Lunze, D. E. Bloom, D. T. Jamison, and D. H. Hamer, "The global burden of neonatal hypothermia: systematic review of a major challenge for newborn survival," (in eng), BMC Med, vol. 11, p. 24, Jan 31 2013, doi: 10.1186/1741-7015-11-24.

[3] D. Askin, "The high-risk newborn and family. Wong's nursing care of infants and children, Wong DL, Hockenberry MJ, and Wilson D. St. Louis: Mosby," ed: Elsevier, 2007.

[4] A. R. Laptook and M. Watkinson, "Temperature management in the delivery room," (in eng), Semin Fetal Neonatal Med, vol. 13, no. 6, pp. 383-91, Dec 2008, doi: 10.1016/j.siny.2008.04.003.

[5] R. Knobel and D. Holditch-Davis, "Thermoregulation and heat loss prevention after birth and during neonatal intensive-care unit stabilization of extremely low-birthweight infants," Adv Neonatal Care, vol. 10, no. 5 Suppl, pp. S7-14, Oct 2010, doi: 10.1097/ANC.0b013e3181ef7de2.

[6] C. Hildebrandt, C. Raschner, and K. Ammer, "An overview of recent application of medical infrared thermography in sports medicine in Austria," Sensors, vol. 10, no. 5, pp. 4700-4715, 2010.

[7] J. I. d. O. Filho and O. d. M. Almeida, "Cloud-based monitoring system and risk management for premature newborns," in 2017 2nd International Symposium on Instrumentation Systems, Circuits and Transducers (INSCIT), 28 Aug.-1 Sept. 2017 2017, pp. 1-6, doi: 10.1109/INSCIT.2017.8103513.

[8] N. Adu-Amankwa and K. Rais-Bahrami, "Evaluation of a wireless cardio respiratory monitor for neonates," Journal of Neonatal-Perinatal Medicine, vol. 4, no. 4, pp. 329-332, 2011.

[9] M. Grubb et al., "Forehead reflectance photoplethysmography to monitor heart rate: preliminary results from neonatal patients," Physiological measurement, vol. 35, no. 5, p. 881, 2014.

[10] L. Scalise, P. Marchionni, I. Ercoli, and E. P. Tomasini, "Simultaneous measurement of respiration and cardiac period in preterm infants by laser Doppler vibrometry," in AIP Conference Proceedings, 2012, vol. 1457, no. 1: AIP, pp. 275-281.

[11] A. C. Kevat, J. Dawson, P. G. Davis, and C. O. F. Kamlin, "Evaluation of a digital stethoscope and smart device technology for assessment of heart rate in the newborn infant," Archives of Disease in ChildhoodFetal and Neonatal Edition, vol. 100, no. 6, pp. F562-F563, 2015.

[12] J. I. d. O. Filho and M. E. d. P. V. Zurita, "Development of NFC TAG for temperature sensing of premature newborns in neonatal incubators," in 2017 2nd International Symposium on Instrumentation Systems, Circuits and Transducers (INSCIT), 28 Aug.-1 Sept. 2017 2017, pp. 14, doi: 10.1109/INSCIT.2017.8103519.
[13] L. Scalise, N. Bernacchia, I. Ercoli, and P. Marchionni, "Heart rate measurement in neonatal patients using a webcamera," in 2012 IEEE International Symposium on Medical Measurements and Applications Proceedings, 2012: IEEE, pp. 1-4.

[14] L. A. Aarts et al., "Non-contact heart rate monitoring utilizing camera photoplethysmography in the neonatal intensive care unit-A pilot study," Early human development, vol. 89, no. 12, pp. 943-948, 2013.

[15] L. K. Mestha, S. Kyal, B. Xu, L. E. Lewis, and V. Kumar, "Towards continuous monitoring of pulse rate in neonatal intensive care unit with a webcam," in 2014 36th Annual International Conference of the IEEE Engineering in Medicine and Biology Society, 2014: IEEE, pp. 38173820.

[16] M. Villarroel et al., "Continuous non-contact vital sign monitoring in neonatal intensive care unit," Healthcare technology letters, vol. 1, no. 3, pp. 87-91, 2014.

[17] S. Nukaya, M. Sugie, Y. Kurihara, T. Hiroyasu, K. Watanabe, and H. Tanaka, "A noninvasive heartbeat, respiration, and body movement monitoring system for neonates," Artificial Life and Robotics, vol. 19, no. 4, pp. 414-419, 2014.

[18] S. Sato et al., "Assessment of a new piezoelectric transducer sensor for noninvasive cardiorespiratory monitoring of newborn infants in the NICU," Neonatology, vol. 98, no. 2, pp. 179-190, 2010.

[19] F. Wang, Y. Zou, M. Tanaka, T. Matsuda, and S. Chonan, "Unconstrained cardiorespiratory monitor for premature infants," International Journal of Applied Electromagnetics and Mechanics, vol. 25, no. 1-4, pp. 469-475, 2007.

[20] L. Atallah et al., "Unobtrusive ECG monitoring in the NICU using a capacitive sensing array," Physiological measurement, vol. 35, no. 5, p. $895,2014$.

[21] T. Kato, A. Ueno, S. Kataoka, H. Hoshino, and Y. Ishiyama, "An application of capacitive electrode for detecting electrocardiogram of neonates and infants," in 2006 International Conference of the IEEE Engineering in Medicine and Biology Society, 2006: IEEE, pp. 916919.

[22] J. V. Kraaijenga, G. J. Hutten, F. H. de Jongh, and A. H. van Kaam, "Transcutaneous electromyography of the diaphragm: A cardiorespiratory monitor for preterm infants," Pediatric pulmonology, vol. 50, no. 9, pp. 889-895, 2015.

[23] R. B. Knobel, B. D. Guenther, and H. E. Rice, "Thermoregulation and thermography in neonatal physiology and disease," Biological research for nursing, vol. 13, no. 3, pp. 274-282, 2011.

[24] A. K. Adams, R. A. Nelson, E. F. Bell, and C. A. Egoavil, "Use of infrared thermographic calorimetry to determine energy expenditure in preterm infants," The American journal of clinical nutrition, vol. 71, no. 4, pp. 969-977, 2000.

[25] A. K. Abbas, K. Heimann, K. Jergus, T. Orlikowsky, and S. Leonhardt, "Neonatal non-contact respiratory monitoring based on real-time infrared thermography," Biomedical engineering online, vol. 10, no. 1, p. 93, 2011 .

[26] K. M. Horns, "Neoteric physiologic and immunologic methods for assessing early-onset neonatal sepsis," The Journal of perinatal \& neonatal nursing, vol. 13, no. 4, pp. 50-66, 2000.

[27] A. K. Saxena and G. H. Willital, "Infrared thermography: experience from a decade of pediatric imaging," European journal of pediatrics, vol. 167, no. 7, pp. 757-764, 2008.

[28] K. Heimann, K. Jergus, A. K. Abbas, N. Heussen, S. Leonhardt, and T. Orlikowsky, "Infrared thermography for detailed registration of thermoregulation in premature infants," Journal of perinatal medicine, vol. 41, no. 5, pp. 613-620, 2013.

[29] S. Ovadia, "Automate the internet with "if this then that"(IFTTT)," Behavioral \& social sciences librarian, vol. 33, no. 4, pp. 208-211, 2014. 